\title{
Translating Simulink Models to Modelica using the Nsp Platform
}

\author{
Jean-Philippe Chancelier ${ }^{1}$ Sébastien Furic ${ }^{2}$ Pierre Weis $^{2}$ \\ ${ }^{1}$ Université Paris-Est, CERMICS (ENPC), 77455 Marne-la-Vallée 2, France, \\ jean-philippe. chancelierdenpc. fr \\ ${ }^{2}$ Inria Paris, 2 rue Simone Iff, 75589 Paris, France \& Université Paris-Est, CERMICS (ENPC), 77455 \\ Marne-la-Vallée 2, France \{sebastien.furic, pierre.weis\} @inria.fr
}

\begin{abstract}
We present a new Simulink (Simulink) to Modelica (Modelica) translation chain embedded into Nsp. Translated models can be edited (original Simulink diagrams are preserved through translation) and simulated. This translation chain makes use of the Simport tool, originally designed to translate Simulink models to Scicos models, and also relies on Modelicac, i.e. Scicos' Modelica companion compiler.

Using some examples, we demonstrate the effectiveness of the translation process and detail some technical aspects of it. This new Nsp feature extends Nsp's simulation capabilities and makes it a reference platform for users looking for means to simulate Simulink models within a Modelica framework. Resulting Modelica code can even be exported to other Modelica compatible tools. Keywords: Nsp; Simulink; Modelica
\end{abstract}

\section{Introduction}

Nsp is a Matlab-like numerical environment which can run the Scicos modeling environment, a Simulink-like block diagram editor and simulator.

From 2003 to 2008, in the course of funded projects SimPA and SimPA2, a Modelica compiler named Modelicac has been developed allowing Scicos to handle genuine Modelica models. This integration of Modelica within Scicos has been the subject of several papers published at the Modelica conference (Nikoukhah and Najafi, 2008; Nikoukhah and Furic, 2009). The purpose of using Modelica is to serve as a high-level description language to extend Scicos expressiveness: Modelica allows users to compose "acausal" models where the original environment forced users to describe their models as block diagrams.

In this paper we focus on a new application of Modelica within Scicos under Nsp, that is as a target language for Simulink model translation.

Several Simulink to Modelica translation tools have already been proposed in the past, we mention in particular Mike Dempsey's Simelica and AdvancedBlocks library (Dempsey, 2003) and Dirk Reusch's Coselica initiative (Reusch). AdvancedBlocks was a fairly complete library of Modelica blocks which allowed users to use Modelica blocks as a one-to-one replacement for Simulink blocks. Up to our knowledge this work remains the most advanced effort in that direction. It is however no longer maintained. In the Scicos software environment, the Coselica library offers signal models to allow users to better exploit Modelica from within Scicos by proposing a large set of Modelica submodels in the same spirit as the standard Modelica library (MSL). Many Simulink-like blocks are also available in Coselica.

The approach presented here differs from Mike Dempsey's and Dirk Reusch's approaches in that translation of original blocks is not attempted on a one-to-one basis. Instead, we use a two steps translation process: the first step translates the Simulink model into an equivalent Scicos native model; the second step translates the Scicos native model to Modelica code.

To handle the initial Simulink to Scicos translation, we use an external tool named Simport: it translates a Simulink model by translating each block in the original Simulink model with one or several blocks of the Scicos native block library, so that original semantics is preserved with a high degree of confidence.

For the second step, we developed a set of Nsp special purpose compilation routines to translate Scicos blocks to genuine Modelica blocks. When a Scicos block has a direct Coselica equivalent, the compilation routine simply emits thre corresponding Coselica block; when there is no Coselica equivalent, the compilation routine generates an entirely new block containing ad-hoc Modelica code to handle the Scicos block behavior. Using this compiletime Modelica code generation and the two steps translation process was the key ideas to fill the huge semantic gap between Simulink and Modelica.

We give in this paper a detailed description of this new translation chain hosted by the Nsp environment.

\section{Involved Tools}

As mentioned above, the translation chain relies on a combination of several tools. We give hereafter a short description of each of them.

\subsection{Nsp, a Programming Environment for Nu- merical Applications}

Nsp (Nsp) is a mature Matlab-like Scientific Software Package developed under the GPL license. Nsp features a high-level, safe imperative programming language with 
automatic memory management. This language can be used interactively, giving users an easy access to efficient numerical routines; It can also be used as a more conventional programming language to extend Nsp's capabilities.

Nsp contains internally a class system with simple inheritance and interface implementation. When used as an interactive computing environment, it comes with online help facilities and an easy access to GUI facilities and graphics.

A large set of libraries are available and it is moreover easy to implement new functionalities. External libraries can also be used: this requires writing some wrapper code (also called interface) to live in harmony with Nsp's internal state. The interface mechanism can be either static or dynamic. By using dynamic functionalities one is able to build toolboxes.

Nsp shares many traits with other Matlab-like Scientific Softwares such as Matlab, Octave, ScilabGtk (ScilabGtk; Campbell et al., 2006), and also with scripting languages such as Python.

The main toolbox used in this work is Scicos that we describe now.

\subsection{Scicos, a Block Diagram Modeler and Sim- ulator}

Scicos (Scicos) is a graphical dynamical system modeler and simulator originally developed in the Metalau project at INRIA, Paris-Rocquencourt center. With Scicos, users can create block diagrams to model and simulate the dynamics of hybrid dynamical systems and compile models into executable code. Scicos is used for signal processing, systems control, queuing systems, and to study physical and biological systems. Extensions allow generation of component-based modeling of electrical and hydraulic circuits using the Modelica language.

We consider in this paper the Scicos/Nsp version of Scicos maintained and developed at ENPC. Scicos/Nsp is a Nsp toolbox and runs in the Nsp environment. Having access to Nsp functions when designing simulation models is of great importance.

Scicos users often needs to use Nsp functions such as those dedicated to filter design for signal processing or controller design in the construction of simulation models. Nsp's programming language can be used for batch processing of multiple simulation tasks, and more generally, models designed by Scicos can be used as functions in Nsp. Nsp's graphical facilities can be used for post processing simulation results. But the integration of Scicos and Nsp goes beyond that. The Scicos editor is entirely written in Nsp's language. This provides many advantages and was in particular of tremendous importance in the current work, indeed: Scicos model data structure is a Nsp structure and thus Scicos models can be programmatically manipulated and build using Nsp scripts. We use this facility in two ways. First to obtain Scicos models from Simulink models, using the fact that the Simport converter produces a Nsp script whose execution in Nsp produces a Scicos model data structure. Second, using Nsp scripts we are able to convert, in a Scicos model data structure, some Scicos blocks to Modelica blocks.

In the conversion process from Simulink to Modelica, the scicos compiler/scheduler also plays a key role. It infers dimensions and types used in the Modelica blocs. This is quite an exciting feature since it gives the possibility to have Modelica blocks for which the associated Modelica model is not a fixed Modelica class but a specific one adapted to specific dimensions and types generated on the fly.

\subsection{Modelicac, a Simple Yet Useful Modelica Compiler}

Development of Modelicac started in 2003 as a joint work between Inria and TNI-Valiosys (now Dassault Systèmes) in the course of the SimPA (SIMulation pour le Procédé et l'Automatique) french funded project. The goal was to make Scicos compatible with a significant subset of the Modelica language in order for users to be able to describe complex hybrid models without having to resort to low-level block diagram descriptions. Indeed, building a block diagram from a physical model requires 1)performing a complete analysis of physical phenomena into play (to determine which elementary blocks to use in the diagram), and 2)determining how data flows between blocks (to connect elementary blocks together). On the other hand, Modelica tools considerably ease physical model construction by automatically analysing the overall structure of physical models described in a much more userfriendly way: familiar physical components (e.g. springs, transistors, hydraulic pumps, etc.) can be used to build models. Translation from this high-level description to low-level data flow is performed automatically in a quite satisfactory way, which frees users from a painful work. Moreover, even slight modifications of physical models may require considerable changes in corresponding blockdiagram descriptions; this is not the case with a high-level description.

In its initial version, Modelicac essentially focussed on the "continuous part" of hybrid models. This mainly comprises differential equations and event-trigerring mechanisms (e.g. , "when equations"). Difference equations were however also be described, although with many restrictions, because the idea was to discourage users from writing discrete equations in Modelica. Indeed, Scicos is primarily a hybrid modelling environment and, in particular, it handles discrete, event-trigered changes, much more robustly than Modelica because of its synchronous roots.

In the course of the SimPA project, the Scicos editor has been extended to enable graphical handling of Modelica, native Scicos, as well as hybrid Modelica-Scicos blocks in the same design (see Figure 1).

This combination of synchronous and Modelica-based features offered enough modelling expressiveness to enable useful libraries to be developed. Coselica is one of these libraries, and is one of the ingredients of our 


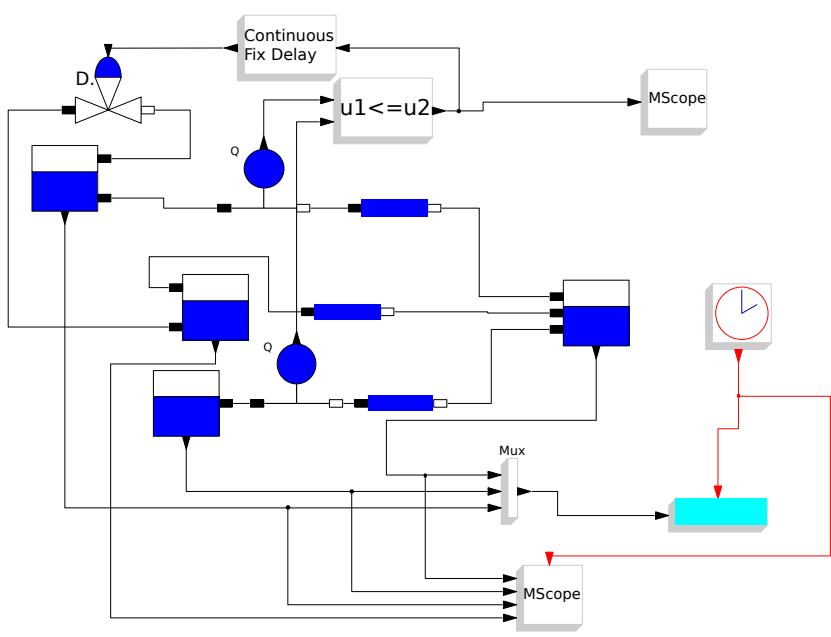

Figure 1. A mixed Scicos-Modelica model as displayed by Scicos's editor

Simulink to Modelica translation chain.

In 2005, the funded project SimPA2 started, having as objective the enhancement of the original Modelicac compiler. Among others, support of multiple-file Modelica libraries and interactive initialization of complex hybrid systems have been added.

As a result, the new Modelicac compiler was able to compete with industrial compilers (it even ranked number two in terms of performance on an industrial thermohydraulic benchmark proposed by EDF in 2009).

Today, the Scicos toolbox with its Modelica-compatible extension is freely available under several environments including Scilab, ScicosLab and Nsp.

\subsection{Simport, a Simulink Model Importer for Scicos}

\subsubsection{Capabilities}

The Simport (Chancelier et al., 2016, 2015) development started in 2007 at Inria: it has now turned into a comprehensive Simulink import assistant for the Scicos and Altair Activate block system modelers: Simport reads a textual representation of a Simulink model (MDL or SLX file format) and generates the corresponding equivalent Scicos model.

Based on compilation techniques, Simport is a fast and reliable translator from Simulink models to Scicos or Altair Activate models.

Simport is a free software distributed with Nsp Sci$\cos$ (Scicos) and Activate (Altair Activate).

\subsubsection{Capabilities}

Simport aims at preserving the original Simulink model semantics: simport performs passes of semantic analysis to explicit the Simulink model meaning and translate it into an equivalent Scicos model.

In any case, the resulting Scicos model preserves the model hierarchy and diagram topology, and the visual aspects of the original model.

Simport also supports both the MDL and SLX formats as input for Simulink models.

\subsubsection{Simulink block translation}

Simport maps Simulink bloks to Scicos blocks using the block translation library. More precisely, each Simulink source block is translated either into

- a single basic block, if there exists an equivalent Scicos block,

- a super-block that implements the Simulink block via a combination of Scicos blocks,

- an empty super-block for user completion, if the Simulink block translation is unsupported

The Simport translation library covers a large subset of Simulink basic blocks, in particular the so-called action blocks.

\subsubsection{Simport generated code}

The Simport back-end translates explicit semantics of Simulink models to concrete code of the Scicos host language (Nsp or Oml). In addition, the concrete code provides the definition of simulation parameters and embeds various outputs to the host language (e.g. Matlab supporting M-files).

\subsubsection{Example}

Given the Simulink model described in Figure 2 and saved as file model.mol.

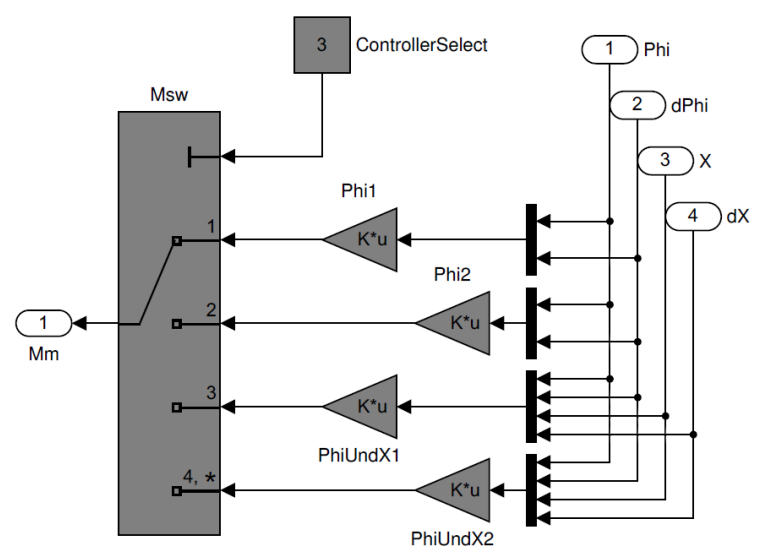

Figure 2. Segway controller as a Simulink model

We translate it into Nsp using the command simport -tl nsp model.mdl. We now get file model.nsp whose execution in Nsp produce build the Scicos model displayed in Figure 3.

\subsubsection{Limitations}

Simport indeed supports a large subset of Simulink basic blocks, but exotic blocks from specific Simulink libraries cannot be translated since they have no Scicos equivalent; 


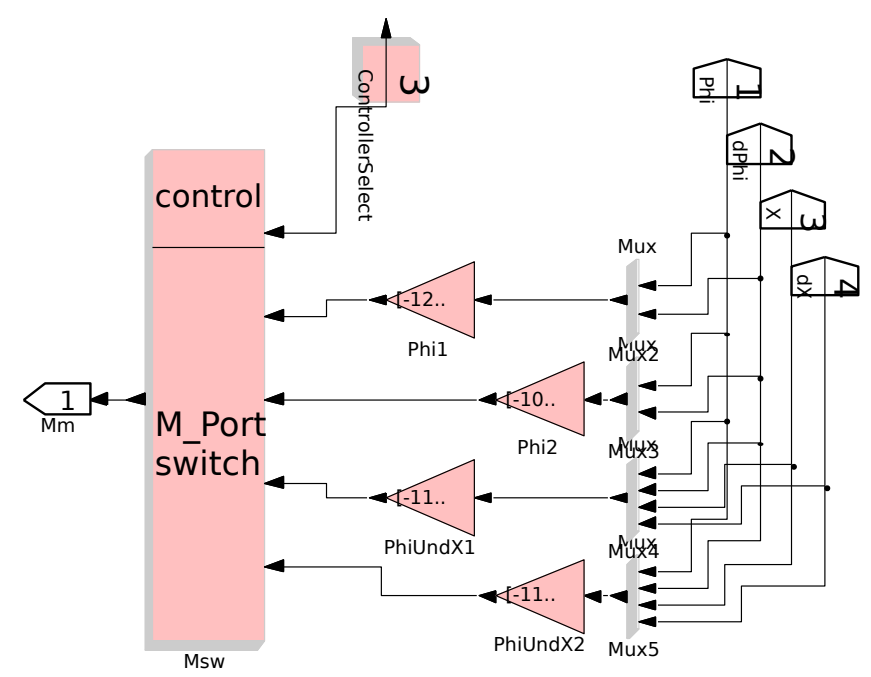

Figure 3. Segway controller as a Scicos model

in such a case, Simport generates an empty Scicos super block to incorporate the mandatory hand written Simulink block translation.

\section{Translation from Simulink to Mod- elica}

The Translation from Simulink to Modelica is implemented as a two step process. First, as already described, using Simport, we translate a Simulink model into a Scicos model. Second, using Nsp scripts we convert the Scicos model into an hybrid Modelica-Scicos model. Conversion is obtained by 1) translation of Scicos blocks to Modelica blocks, and 2) addition in the model of converters along the links which connect Scicos Blocks to ModelicaScicos blocks. The hybrid Modelica-Scicos models can be edited and simulated in Scicos editor; thus, even if during the Translation process we cannot obtain a full Modelica model ${ }^{1}$, the resulting hybrid model may still be used for simulation because users have the possibility to complete untranslated parts thanks to the Scicos editor.

Figure 4 is a Scicos model simulating the Lorenz dynamical system. The same model after conversion to Modelica is shown in Figure 5. As it can be seen in Figure 5 the Scopes are not translated to Modelica blocks and converters from Modelica signals to Scicos signals are inserted in the links connected to the entry ports of scopes.

When converters are available, Scicos blocks are replaced by Modelica blocks as a one-to-one process. We have developed a specific library of Modelica blocks to ease the replacement. For example Scicos integrator blocks are replaced by MB_Integral Modelica blocks. For some translation we rely on the already available library Coselica (Reusch), but for many blocks a direct Coselica translation fails because of size lim-

\footnotetext{
${ }^{1}$ In case some blocks are unknown to Simport. Indeed, Simulink blocks are black boxes, so Simport cannot translate blocks or combinations of blocks that are not already described in its translation tables.
}

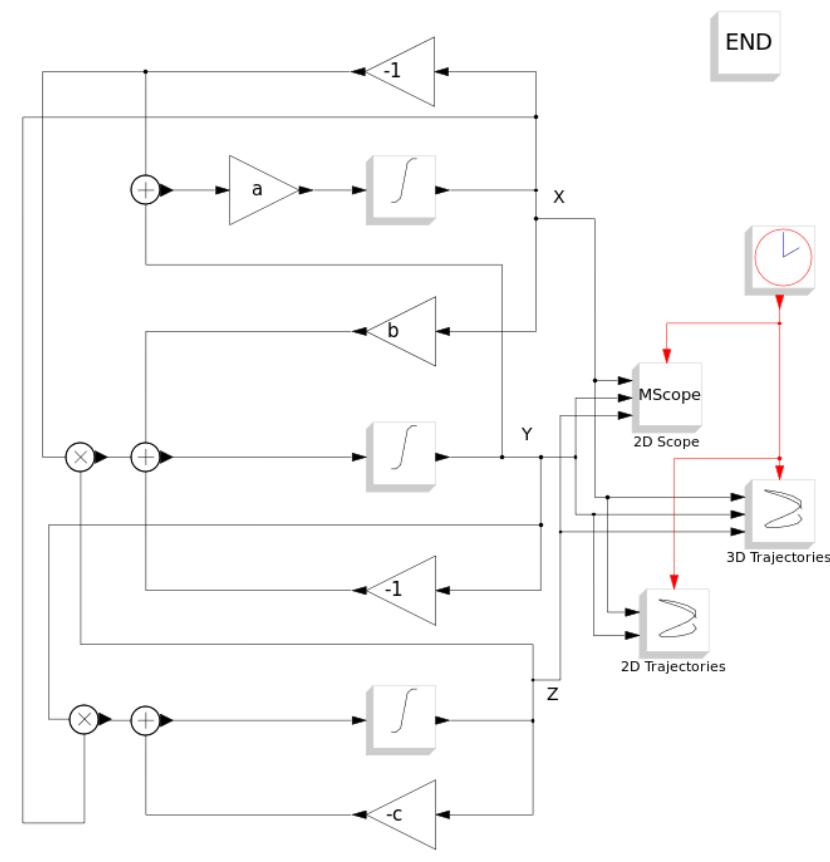

Figure 4. A Scicos model as displayed by Scicos's editor

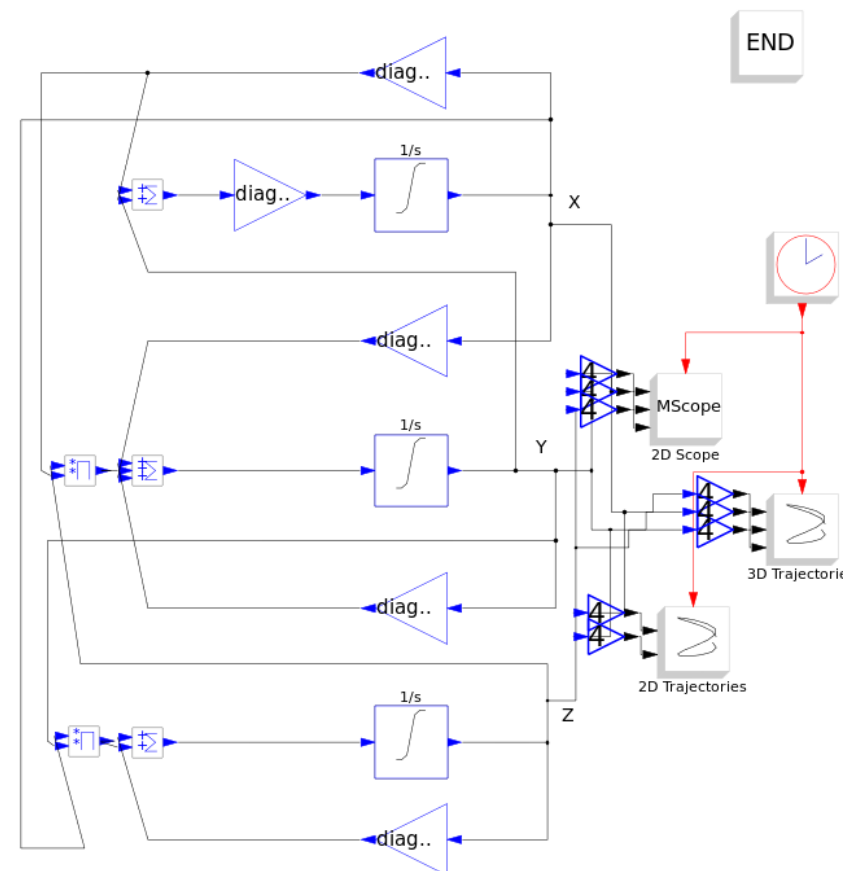

Figure 5. Scicos model after Modelica conversion as displayed by Scicos's editor 
itations of Coselica blocks. For example the Coselica Integrator is limited to 1-dimensional signals while the Scicos INTEGRAL_m block may have $n$-dimensional entries. One way to encompass that difficulty is to generate super blocks for enabling $n$-dimensional block operations from 1-dimensional basic blocks (See Figure 7 for an example with adder). We have chosen this approach for the converter blocks (See Figure 6) as explained below, but we also implemented specific blocks to deal with n-dimensional signals. For example, the MB_Integral block is a special purpose Modelica-Scicos block which produces at compile time a new Modelica model for each instance of the block in a specific model. As an example, in Figure 5 each MB_Integral Modelica block integrate a 4-dimensional variable without saturation and thus the generated code will be given by

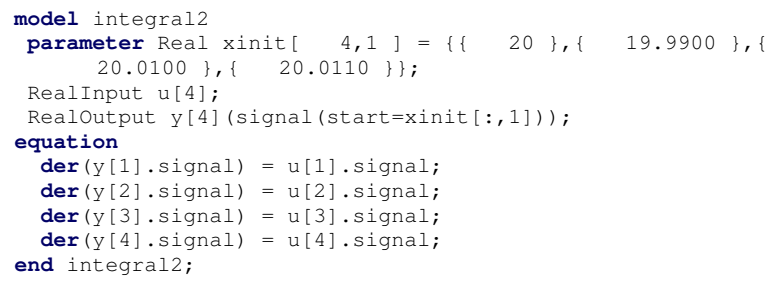

Most of the one-to-one block conversion follows the same mechanism. Building a library of Modelica-Scicos blocks is an on-going work and for the time being it only contains about 20 blocks. Indeed, this Library can also be used to directly build models in the Scicos editor, it complements the set of Modelica block available in Scicos giving access to Modelica counterpart of known Scicos blocks.

The one-to-one block conversion is in fact also a multistep process. We proceed as follows.

First block-to-block conversions are performed but converted Modelica-Scicos blocs are not fully usable because they lack local information (for example the final matrix sizes are unknown at first step). Notice that this first step requires Nsp evaluation of block parameters since they may be used to infer types and dimensions. For example the sizes of a Gain block parameter gives the input/output port sizes of the block, except when the parameter size if 1. But in order to obtain the sizes of a given Gain block parameter we need to evaluate Nsp expressions, since parameters can be given through context (produced by Simport from Matlab companion files).

In a second step, links are modified and converters are inserted where appropriate. Notice however that converters sizes are also unknown.

In a third step, sizes and types are obtained by calling the Scicos model compiler. However, since the Scicos model compiler only infers types and dimension for Scicos blocks this step requires a hidden conversion of the hybrid Modelica-Scicos model into a pure Scicos model before trying to infer sizes and types. When sizes and types are inferred for a Modelica-Scicos block, its internal Modelica code can be generated. The code is thus consistent with respect to sizes, types and parameters.

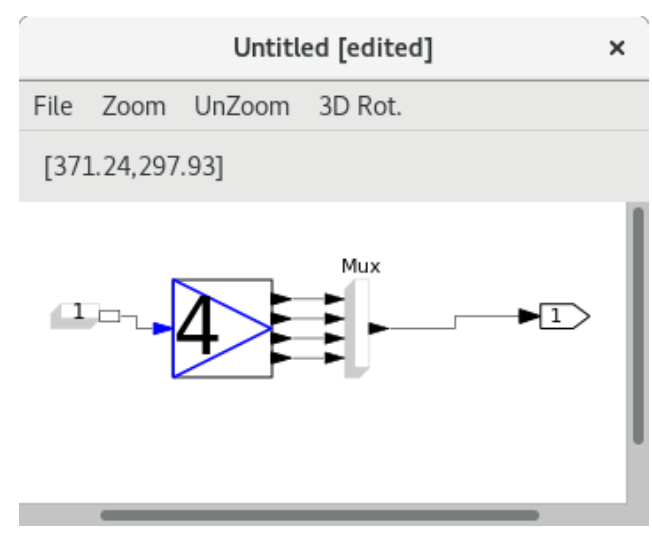

Figure 6. Scicos internal model of a 4-dimensional Modelica to Scicos converter

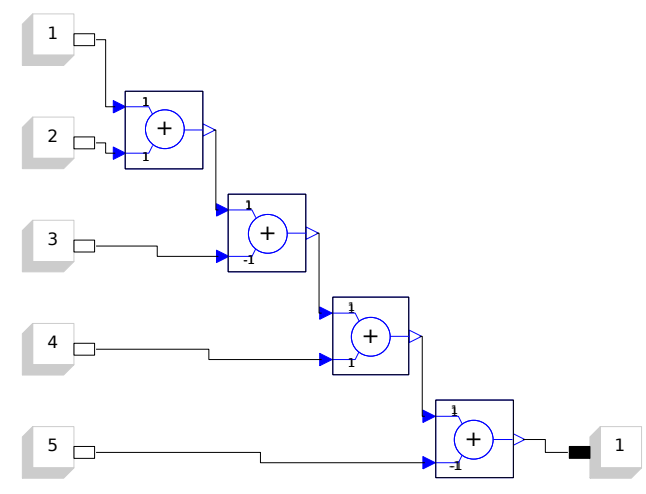

Figure 7. Scicos internal model of a generated 5-dimensional addition block

The fact that models can be manipulated and generated programmatically is also used in the conversion process. We illustrate this point by describing more precisely $\mathrm{MB} \_\mathrm{MO} 2 \mathrm{Sn}$ the block used to convert Modelica signals to Scicos Signals. The communication between Scicos and Modelica can only be realized using scalar links (for historical reasons, not because of limitations of any of the languages), thus to have converters on links which transfer n-dimensional signals we have implemented a block named $\mathrm{MB} \_\mathrm{MO} 2 \mathrm{Sn}$ as a super-block. That is, the MB_MO2 Sn block contains a model and this model is generated dynamically when the link signal size is known. We give in Figure 6 the internal model of a 4-dimensional Modelica to Scicos converter as used in the model displayed in Figure 5. It contains four 1-dimensional Modelica to Scicos converters. To illustrate the possibility to generate models by program, we give in Figure 7 an example of a model which performs a 5-dimensional addition of Modelica signals. Implementing a $n$-dimensional adder block could be implemented that way, even if we chose to directly embed the Modelica $n$-dimensional adder block in a unique block.

As already pointed above, during the conversion from Scicos models to Modelica-Scicos models, inferring types 


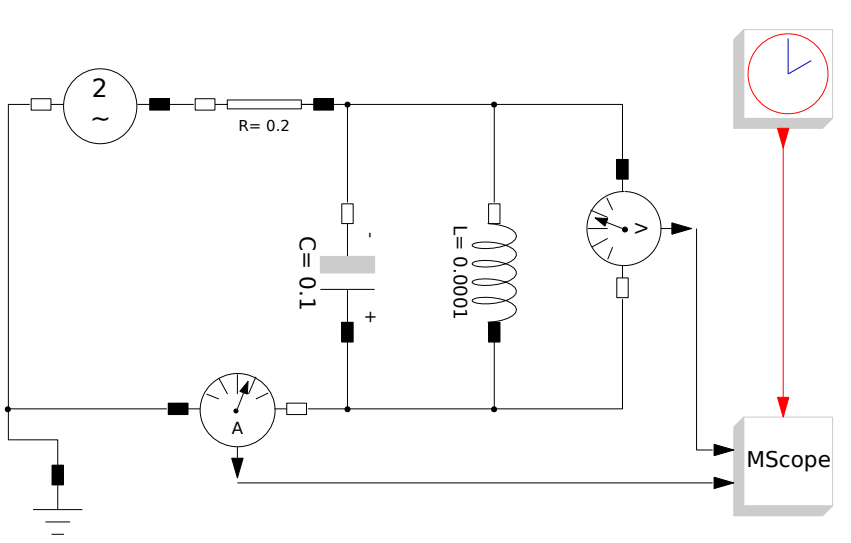

Figure 8. A mixed Scicos-Modelica model of a RLC circuit

and sizes is of utmost importance and it partially relies on Nsp block parameter and context expression evaluation. This is mostly why the conversion cannot completely be performed by Simport. Indeed, inferring types and sizes could have been implemented directly in Simport, if evaluation of Matlab expression were not required in the process.

\subsection{Translation from Modelica to $\mathbf{C}$}

Modelica source code is translated to $\mathrm{C}$ thanks to the Modelicac compiler. The idea is as follow. Once the model is being run by the user, Scicos gathers all the blocks whose execution semantics is described by means of Modelica code into a unique Modelica program whose source code is given to Modelicac. This program is actually a highlevel description of the Modelica part of the model. The following listing illustrates what such a description looks like. It contains the Modelica description generated by Scicos for the model of Figure 8:

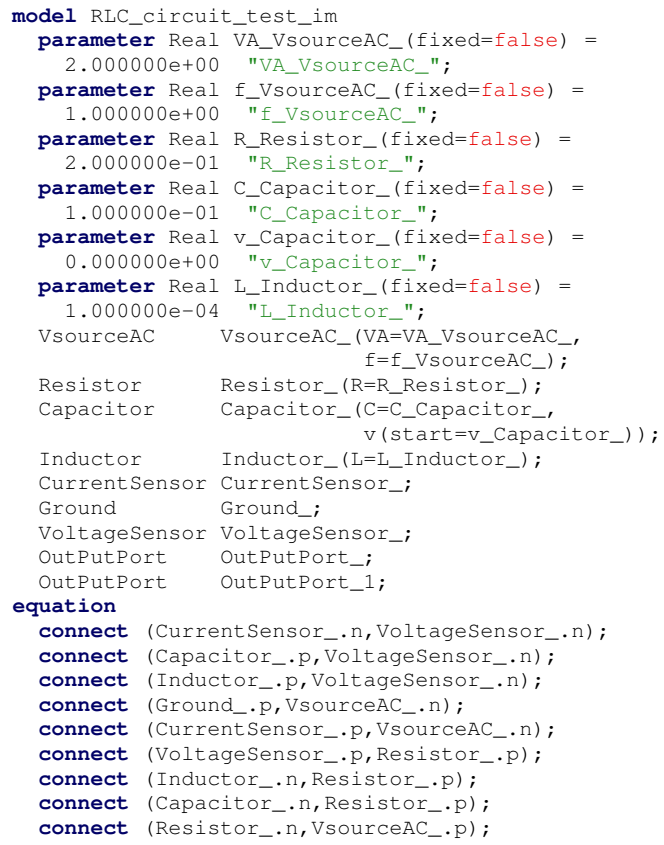

Modelica programs generated by Scicos contain five (possibly empty) sections declaring respectively:

- the parameters of the model,

- the components appearing in the model (i.e. Modelica "blocks" used to build the model),

- the connectors to and from the Scicos world (declared as InPutPorts and OutPutPorts),

- the connection equations (corresponding to links between components of the model, introduced by means of the connect keyword), and

- the correspondence between some Scicos ports and some Modelica connectors used to exchange information between both worlds (introduced by means of an equal sign).

From such Modelica programs Modelicac generates native, C-based Scicos blocks. It starts by resolving the names appearing in the Modelica description and instantiates required classes (found in libraries) to form the set of all equations governing the dynamics of the Modelica part of the model. It then flattens the structure of the Modelica model, simplifies equations, and generates $\mathrm{C}$ code. The following listing is the result of calling Modelicac with previous Modelica code:

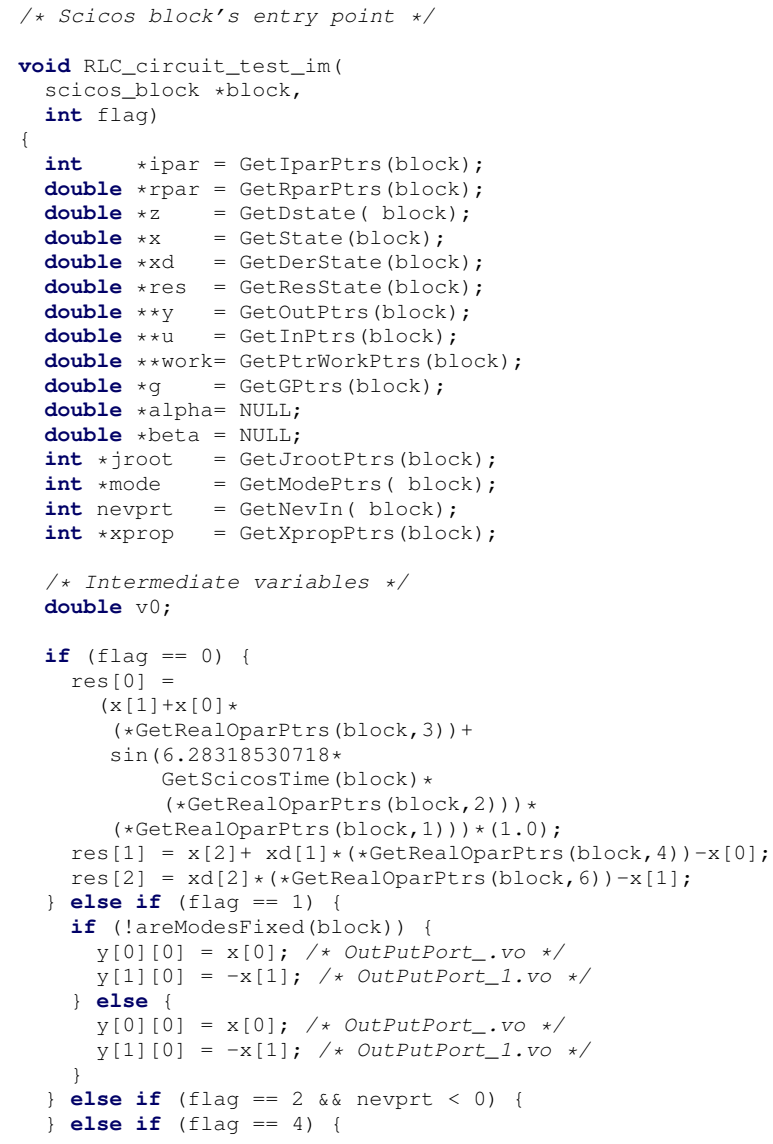




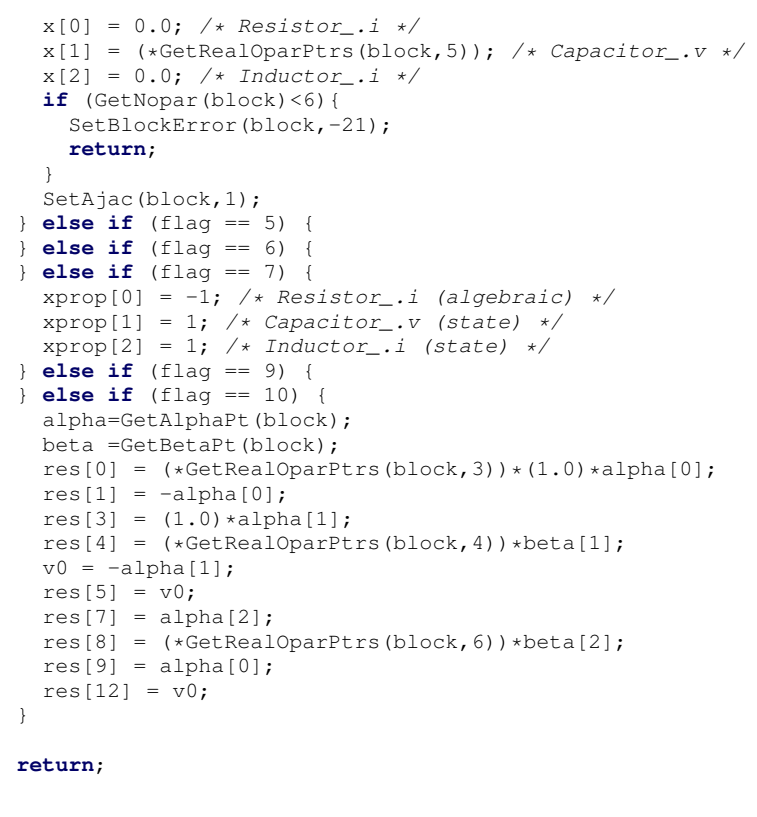

Finally, a new native Scicos block running the generated C code is created by Scicos and connected to the Scicos part of the original model in place of the Modelica blocks. Scicos then performs the simulation of the resulting model.

\section{Working with Acausal Models}

Although supported Simulink models are limited to explicit input-output blocks, one should not conclude that our tool chain only deals with "causal" modeling. Indeed, Scicos provides a powerful editor (written in Nsp's language) by means of which imported Simulink models can be graphically connected to Modelica "acausal" models. This allows, for instance, control models written in Simulink to be imported in Nsp and connected to Modelica models (handled by Modelicac). Complete models featuring both "causal" and "acausal" aspects can then be simulated and possibly exported as Modelica code (Figure 1 and Figure 8 show examples of Modelica "acausal" models with control and displays modeled as block-diagrams).

\section{Conclusion and Future Work}

In this paper we have shown that NSP can be used as a powerful environment to translate, possibly edit, and simulate some Simulink models. The tool chain comprises two external tools, namely Simport and Modelicac, and the Scicos toolbox with its hybrid Scicos-Modelica library Coselica.

This addition to NSP allows users to simulate many Simulink models with few changes, if any. Moreover, original models can be edited after translation, and possibly connected to native Scicos models, or to Modelica models. This opens many interesting perpectives for users willing to run heterogeneous models at a very high level, using the graphical editor provided by Scicos as the sole GUI.
Several enhancements can be made to this preliminary work. The most significant enhancement would probably consists in enriching the Scicos to Modelica translation table, to allow more Simulink models to be translated automatically.

\section{Acknowledgements}

We would like to thank Ramine Nikoukhah from Altair for his considerable help in the design and implementation of the tools we used, in particular Scicos of course, but also Modelicac and Simport.

\section{References}

Altair Activate. Multi-Disciplinary System Simulation. URL https://solidthinking.com/product/ activate.

Stephen Campbell, Jean-Philippe Chancelier, and Ramine Nikoukhah. Modeling and Simulation in Scilab/Scicos. Springer, 2006. ISBN: 978-0-387-27802-5.

Jean-Philippe Chancelier, Fraņois Delebecque, Clément Franchini, Ramine Nikoukhah, and Pierre Weis. Simport: A Simulink Model Importer for Scicos. In The 3rd International Workshop on Simulation at the System Level, École Normale Supérieure de Cachan, France, 2015.

Jean-Philippe Chancelier, Franois Delebecque, Clément Franchini, Ramine Nikoukhah, and Pierre Weis. Simport. In ISC'2016 Bucharest, 2016.

Mike Dempsey. Automatic translation of simulink models into modelica using simelica and the advancedblocks library. In Proceedings of the 3rd International Modelica Conference, Linköping, Sweden, pages 115-124, 2003.

Modelica. The modelica language specification. URL https : //modelica.org/documents.

Ramine Nikoukhah and Sébastien Furic. Towards a full integration of modelica models in the scicos environment. In Proceedings of the 7th International Modelica Conference, Como, Italy, pages 641-645, 2009.

Ramine Nikoukhah and Masoud Najafi. Initialization of modelica models in scicos. In Proceedings of the 6th International Modelica Conference, Bielefeld, Germany, pages 3746, 2008.

Nsp. A Numerical computing environment (GPL). URL http://cermics.enpc.fr/ jpc/nsp-tiddly/ mine.html.

Dirk Reusch. Coselica toolbox für scicoslab. URL http: //www.kybdr. de/software\#coselica_ toolbox_fuer_scicoslab.

Scicos. Block diagram modeler/simulator. URL http: / / www.scicos.org/.

ScilabGtk. Gtk+ version of Scilab. URL http://www . scilabgtk.org.

Simulink. System modeling and simulation. URL https:// www. mathworks. com/products/simulink. html. 
